\title{
Identifying the determinants of response to MDM2 inhibition
}

\author{
Anne Y. Saiki ${ }^{1}$, Sean Caenepeel $^{1}$, Elissa Cosgrove ${ }^{2,5}$, Cheng Su$^{3}$, \\ Michael Boedigheimer ${ }^{4}$, Jonathan D. Oliner ${ }^{1}$ \\ ${ }^{1}$ Oncology Research, Amgen, Inc., Thousand Oaks, California, USA \\ ${ }^{2}$ Genome Analysis Unit, Amgen, Inc., South San Francisco, California, USA \\ ${ }^{3}$ Biostatistics, Amgen, Inc., Seattle, Washington, USA \\ ${ }^{4}$ Molecular Sciences, Amgen, Inc., Thousand Oaks, California, USA \\ ${ }^{5}$ Department of Molecular Biology and Genetics, Cornell University, Ithaca, New York, USA
}

Correspondence to:

Jonathan D. Oliner, e-mail: jonoliner@hotmail.com

Keywords: TP53, MDM2, amplification

Received: October 28, $2014 \quad$ Accepted: January 08, 2015 Published: February 03, 2015

\section{ABSTRACT}

Previous reports have provided evidence that p53 mutation is a strong negative predictor of response to MDM2 inhibitors. However, this correlation is not absolute, as many p53Mutant cell lines have been reported to respond to MDM2 inhibition, while many p5 $3^{\mathrm{WT}}$ cell lines have been shown not to respond. To better understand the nature of these exceptions, we screened a panel of $\mathbf{2 6 0}$ cell lines and noted similar discrepancies. However, upon extensive curation of this panel, these apparent exceptions could be eliminated, revealing a perfect correlation between p53 mutational status and MDM2 inhibitor responsiveness. It has been suggested that the MDM2-amplified subset of p53 ${ }^{\mathrm{WT}}$ tumors might be particularly sensitive to MDM2 inhibition. To facilitate clinical testing of this hypothesis, we identified a rationally derived copy number cutoff for assignment of functionally relevant MDM2 amplification. Applying this cutoff resulted in a pan-cancer MDM2 amplification rate far lower than previously published.

\section{INTRODUCTION}

The tumor suppressor protein $\mathrm{p} 53$ plays a critical role in protecting cells from various stresses, such as DNA damage and hypoxia [1]. In response to these triggers, activated p 53 upregulates the transcription of a host of genes involved in cell cycle arrest, apoptosis, DNA repair, and senescence [2]. Tumor cells are under constant cellular stress, and there is a selective survival advantage for such cells to disrupt the p53 pathway. Indeed, inactivation of $\mathrm{p} 53$ by mutation and/or loss occurs in approximately $50 \%$ of human tumors [2].

MDM2, another key member of the pathway, negatively regulates 553 by 1 ) binding to and blocking the transcriptional activation domain of $\mathrm{p} 53,2$ ) exporting p53 from the nucleus to the cytoplasm, and 3) promoting the degradation of $p 53$ through its E3 ubiquitin ligase activity [3]. Gene amplification of $M D M 2$ occurs at high frequency in sarcomas and at low frequency in cancers of the brain, bladder, stomach, lung, skin, and breast [4].

The MDM2-p53 protein-protein interaction can be disrupted by small molecule inhibitors which occupy the p53 binding pocket of MDM2, leading to the stabilization of p53 and activation of the pathway [5]. Several MDM2 inhibitors are currently in clinical development $[6,7]$. In order to better understand which patients might realize the greatest benefit from MDM2 inhibitor treatment, we set out to identify the determinants of sensitivity and/or resistance by screening a broad panel of tumor cell lines. Additionally, we mined data generated by the TCGA Research Network [4] to rationally define parameters for clinical testing of the hypothesis that MDM2 amplification might enhance sensitivity of $\mathrm{p} 53^{\mathrm{WT}}$ tumors to MDM2 inhibition.

\section{RESULTS}

Sensitivity profiling of MDM2 inhibitor AMGMDS3 in a panel of tumor cell lines

As a first step towards identifying the determinants of sensitivity to MDM2 inhibition, a panel of 260 human tumor cell lines of diverse tissue origins was screened in a 72-hour cell proliferation assay. The effect of MDM2 inhibitor AMGMDS3 (Figure S1) on cell proliferation was 
determined by relative cell count as measured by nuclear staining, with $\mathrm{IC}_{50}$ values ranging from $0.01 \mu \mathrm{M}$ to $>50$ $\mu \mathrm{M}$ (Figure 1A, Table S1). In agreement with previous findings (plotted from published data in Figure $1 \mathrm{~B}-1 \mathrm{C}$; $[8,9])$, sensitivity to MDM2 inhibition was highly correlated with p53 mutational status. This was a predictable result, as p53 mutations prevent p53 from activating transcriptional targets responsible for inducing cell cycle arrest and apoptosis. However, the correlation between p53 mutational status and sensitivity was not universal: some $\mathrm{p} 53^{\text {Mutant }}$ cell lines appeared to be sensitive to MDM2 inhibition, while some $\mathrm{p} 53^{\mathrm{WT}}$ cell lines appeared to be insensitive. We suspected that some of these discrepancies might be related to misannotation or other confounding factors, and we therefore set out to comprehensively curate this cell line panel.

Twenty-six cell lines were removed from the dataset due to the poor growth characteristics of untreated cultures or high coefficient of variance between replicate untreated samples (Table S1). To authenticate the remaining cell lines, we extracted genomic DNA and performed genome-wide SNP analysis. We compared the resulting SNP profiles with those from the GlaxoSmithKline data repository (http:/www.cabig.nci.nih.gov/community/ caArray_GSKdata/; [10]) and Wellcome Trust Sanger Institute Cancer Genome Project (http://www.sanger. ac.uk/genetics/CGP; [11]). We determined that 5 cell lines had been misidentified and 22 cell lines were synonymous with one or more cell lines already represented in the panel (Table S1). These cell lines were excluded from further analysis.

\section{Functional inactivation of wildtype p53 by viral genes can affect proper assignment of p53 mutational status}

The E6 protein from human papillomavirus (HPV) is known to bind p53 and promote its degradation via the ubiquitin pathway [12]. Proteins from DNA polyoma viruses SV40 (TAg) and adenovirus (E1B) also associate with p53 to form stable complexes (reviewed in [13]). While these virally-infected cell lines typically possess wildtype p53 alleles, they lack functional p53 protein. To determine whether any lines in the panel harbored viral DNA, we used PCR to screen their genomic DNA for HPV E6 (high-risk types 16, 18, 31, 33, and 45), SV40 large $\mathrm{T}$ antigen, and adenovirus E1B sequences (Table S2). Six lines were found to contain viral E6 DNA sequences from HPV16 (DoTc2 4510, SiHa, and engineered line RKO E6 [14]) or HPV18 (C-4 I, C-4 II, and HeLa). In addition, SV40 large $\mathrm{T}$ antigen sequence was detected in BPH1 and NCI-H295R. Adenovirus E1B sequence was not detected in any of the cell lines. All cell lines positive for the presence of viral DNA sequence were excluded from further analysis (Table S1).

\section{Genomic sequencing alone can determine p53 mutational status for many, but not all, cell lines}

In order to establish the p53 status of the cell lines in the panel, exons 2-11 of the TP53 gene, along with portions of the neighboring introns, were sequenced from genomic DNA samples extracted from each of the cell lines tested, with the exception of VCAP (sample unavailable). TP53 sequence was determined for nearly all of the cell lines; the cell lines that failed sequencing for a subset of exons were annotated as deletion mutants (Table $\mathrm{S} 1)$. Additionally, twenty-five cell lines were identified as $\mathrm{p} 53^{\text {Mutant }} / \mathrm{p} 53^{\text {WT }}$ heterozygotes by sequencing (Table S1) and were excluded from the dataset to avoid ambiguity.

We utilized the IARC TP53 database to evaluate each of the sequenced missense mutations based on the comprehensive functional analysis of p53 mutant proteins performed by Kato et al. [15]. Nearly all of the mutations that we identified were annotated as inactivating. However, one amino acid substitution, Q331R in cell line 22Rv1, was shown by Kato et al. to be transcriptionally active. Therefore, this cell line was annotated as functionally wild-type and retained for further analysis.

As part of the cell panel curation, we searched for drug sensitivity correlates that might reveal previously unrecognized confounders to the stratification analysis. Strikingly, we observed that the four least responsive p53 ${ }^{\mathrm{WT}}$ cell lines (CAPAN-2, MDA-MB-453, MG-63, and NCI-H82) also displayed the lowest expression levels of TP53 transcript (Figure 2). Indeed, these 4 cell lines occupied a spatially distinct cluster in the plots of sensitivity vs. TP53 expression. To further investigate p53 expression in these cell lines, immunoblot analysis was performed following 24 hours of treatment with MDM2 inhibitor AMG 232 [6]. HCT116, a p53 ${ }^{\mathrm{WT}}$ cell line that is sensitive to MDM2 inhibition, was used as a control in these experiments. As expected, AMG 232 treatment of HCT116 cells resulted in upregulation of MDM2 and p21 expression, as well as accumulation of p53 (Figure 3A). No such upregulation was seen in the other 4 cell lines, suggesting that p53 was non-functional in these lines. Additionally, in MDA-MB-453 cells, a band which migrated faster than the control was detected, indicative of a truncated mutant $\mathrm{p} 53$ protein, consistent with previously reported data (Figure 3A; [16]).

To investigate the mechanism(s) underlying the apparent p53 inactivation in CAPAN-2, MG-63, and NCI-H82, we sequenced TP53 cDNA from these cell lines to determine whether or not there were mutations present in the transcript. TP53 cDNA could not be isolated from MG-63 (data not shown). Since there were previously published data for MG-63 which demonstrated that TP53 is rearranged within the first intron [17], this line was annotated as mutant. For CAPAN-2 and NCI-H82, TP53 cDNA clones were generated and sequenced. Sequence 


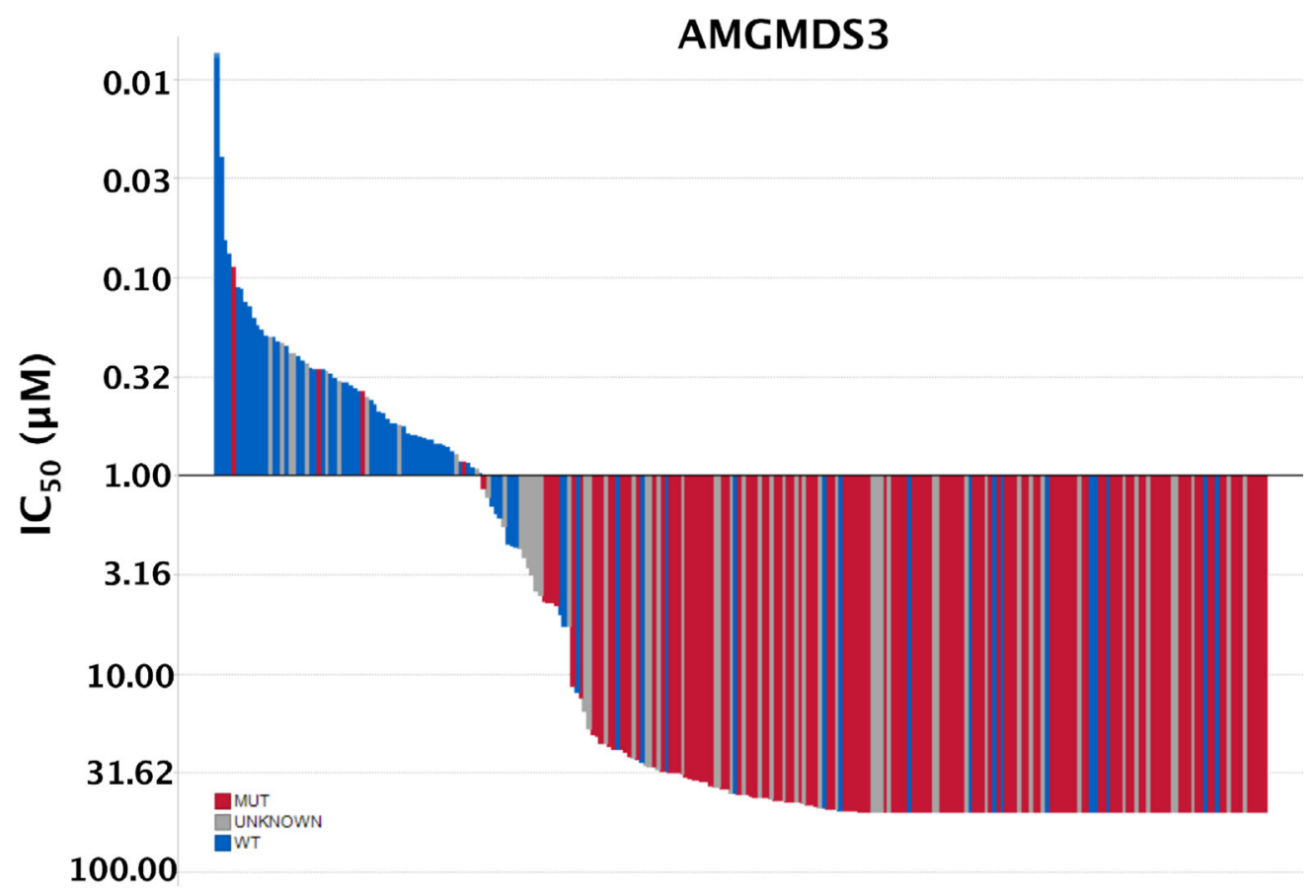

Cell Lines (260)

B

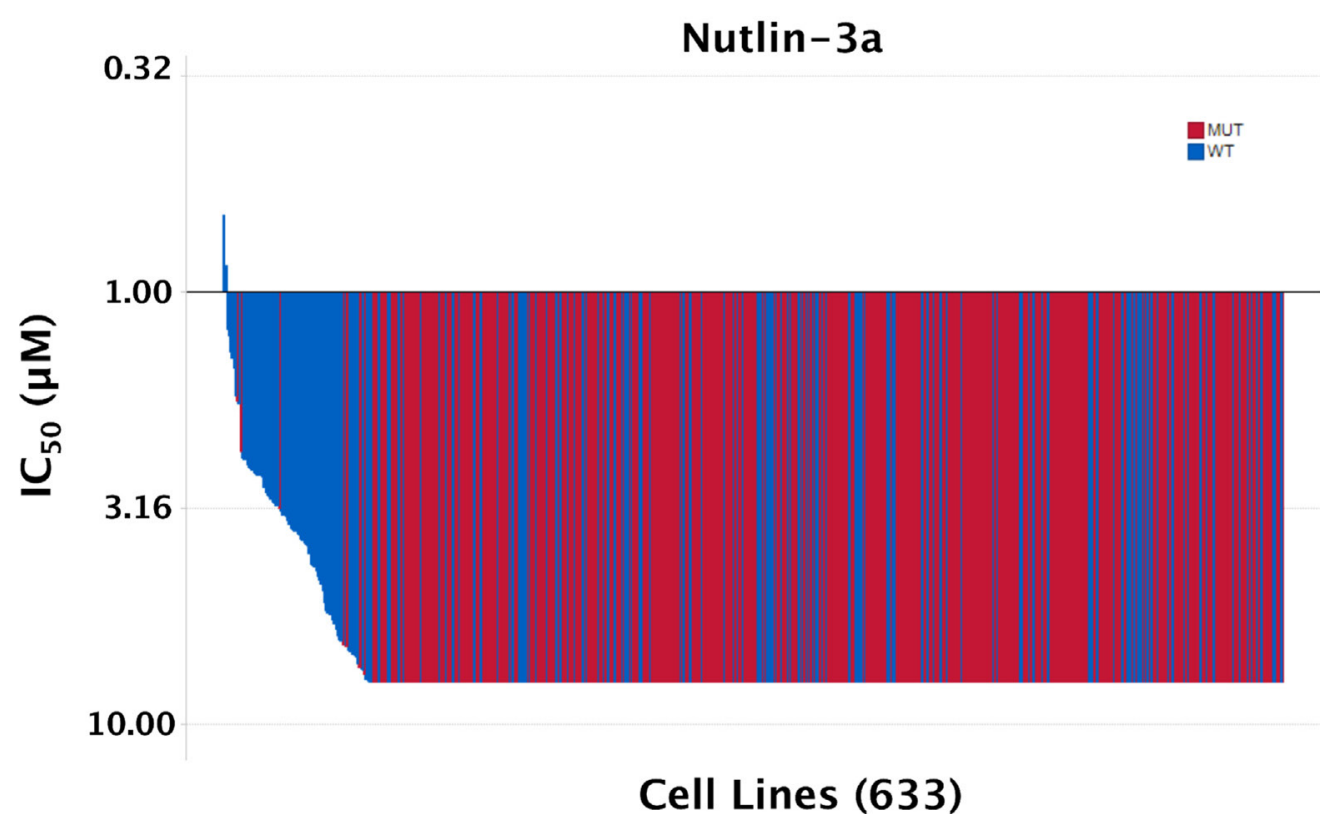

Figure 1: Sensitivity to MDM2 inhibition highly correlates with TP53 mutational status. (A) The sensitivity to AMGMDS3 was profiled across a panel of 260 tumor cell lines in a 72-hour cell proliferation assay. The TP53 mutational status of each cell line was annotated according to the data available in COSMIC (v44 release), http://www.sanger.ac.uk/cosmic [11, 28]. Similar representations of previously published nutlin-3a sensitivity data from (B) Garnett et al. [8] and (Continued) 
C

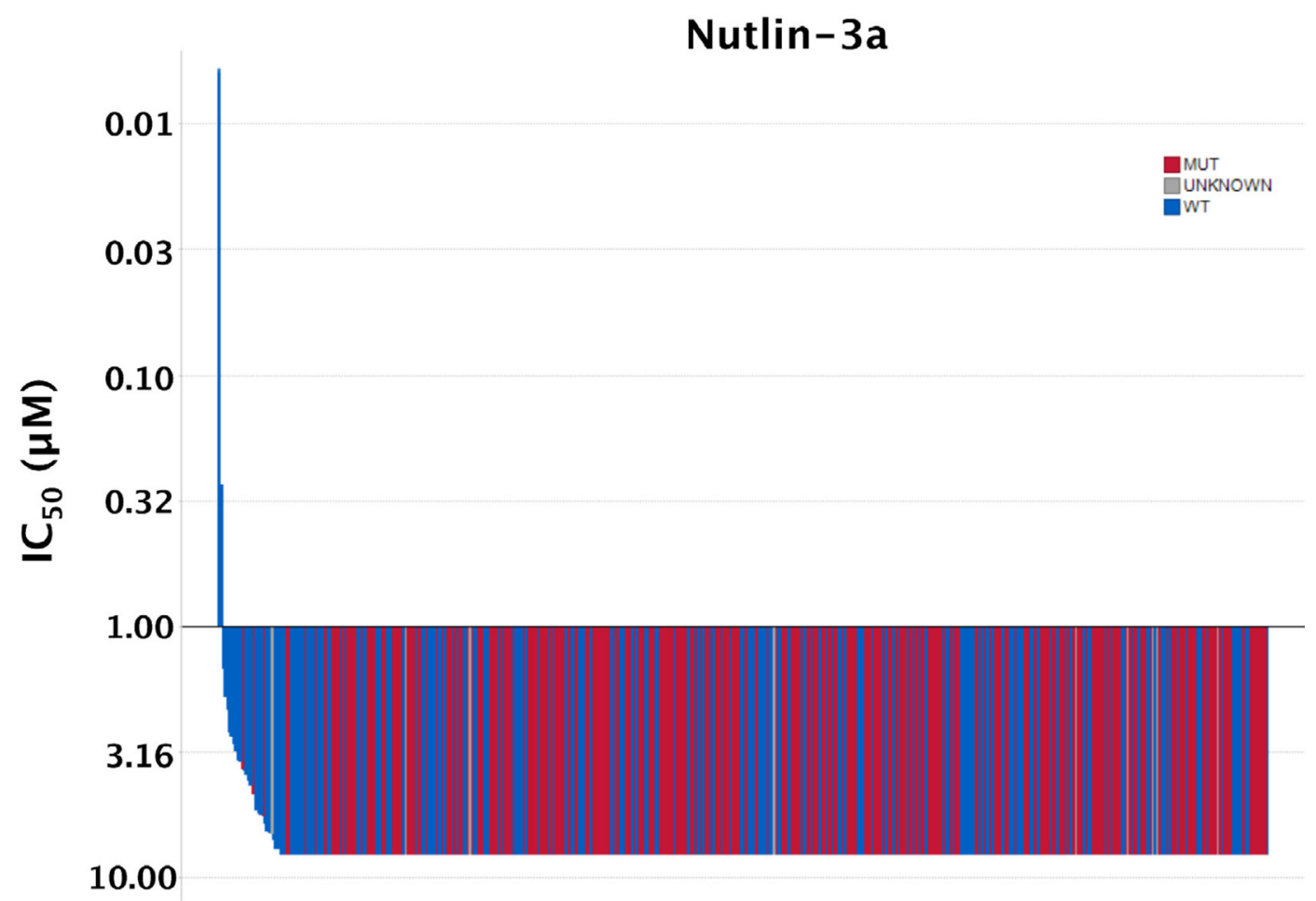

Figure 1: (Continued) (C) Barretina et al. [9] have been shown for comparison. The highest concentration of nutlin-3a tested by Garnett et al. and Barretina et al. was $8 \mu \mathrm{M}$.

A

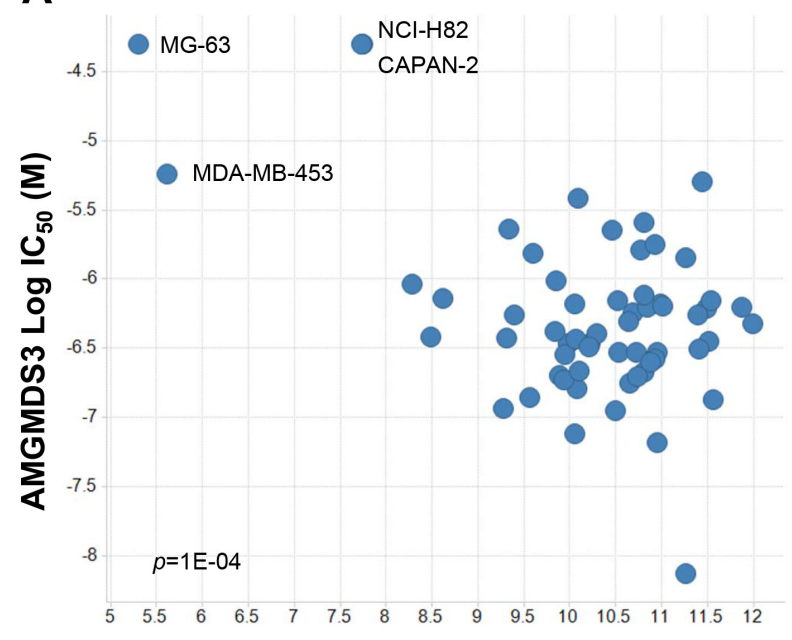

TP53 expression (201746_at)

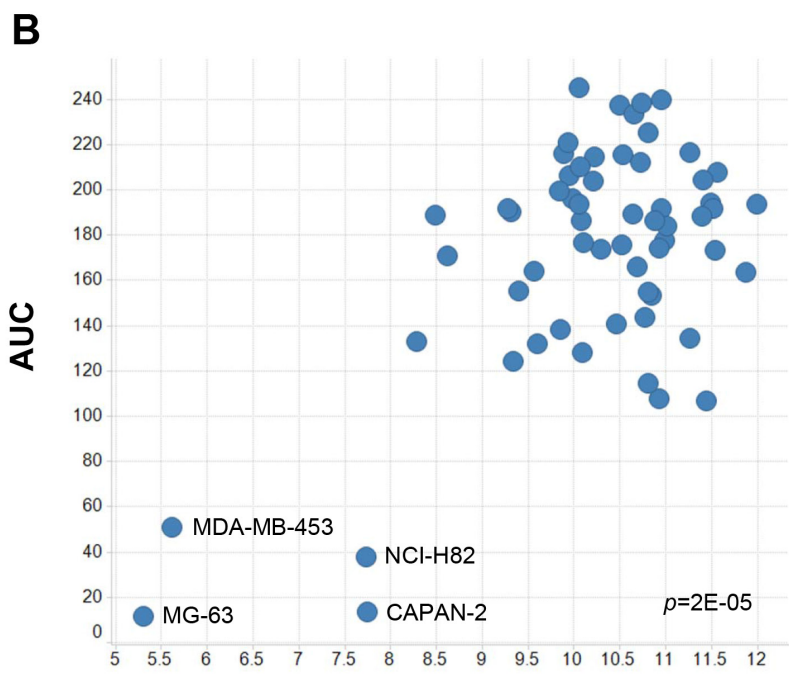

TP53 expression (201746_at)

Figure 2: Preliminary linear regression association analysis indicates that low p53 expression correlates with insensitivity to MDM2 inhibition. Scatter plots of (A) AMGMDS3 cell proliferation $\mathrm{IC}_{50}(p=1 \mathrm{E}-04)$ or (B) integrated area under the dose response curve ( $p=2 \mathrm{E}-05)$ versus TP53 gene expression (201746_at; [10]) for the 62 cell lines with wildtype TP53 genomic sequence profiled in this study. Further analysis revealed that CAPAN-2, MDA-MB-453, MG-63, and NCI-H82 were, in fact, p53 $3^{\text {Mutant }}$ cell lines (see Figure 3). 

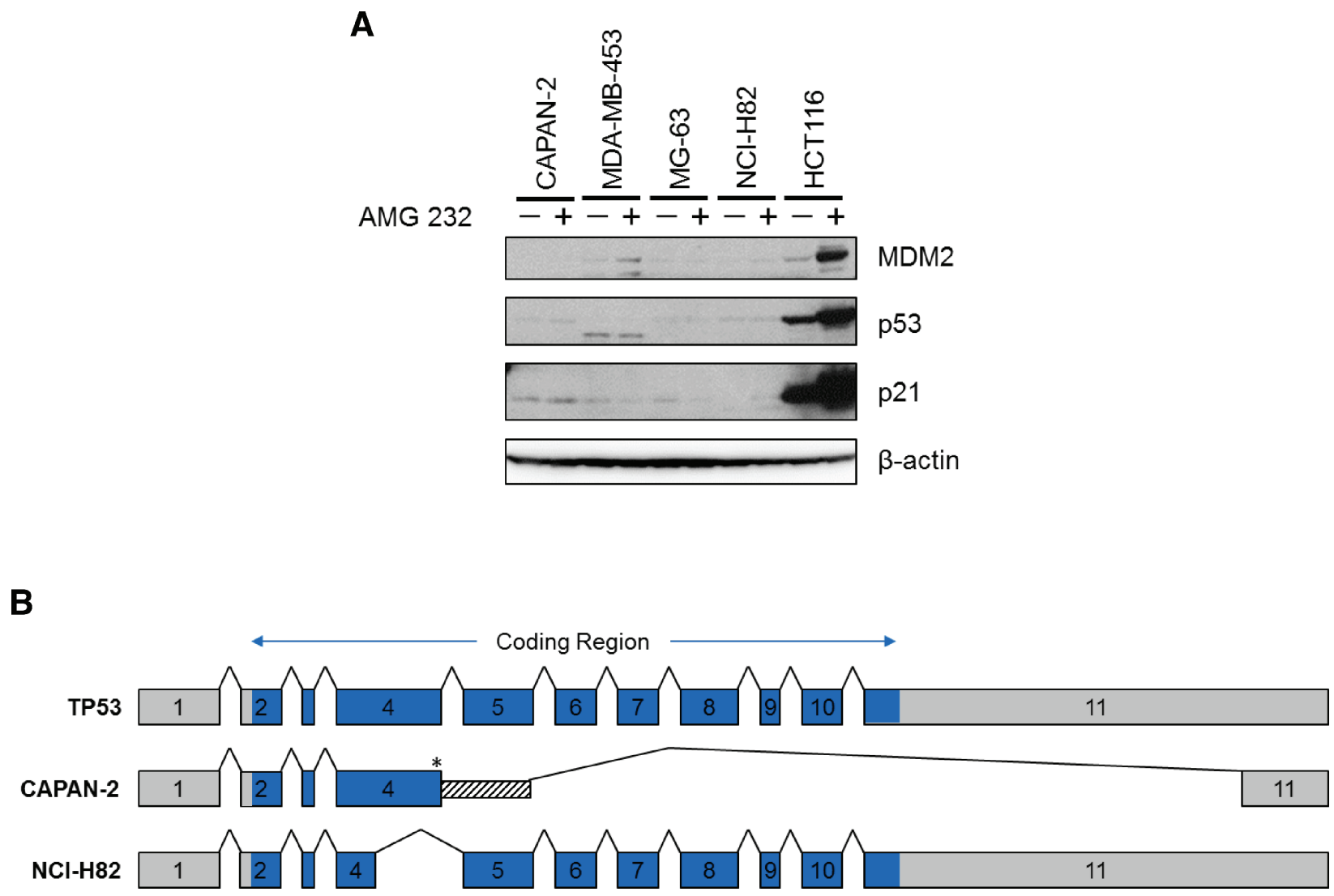

Figure 3: Four insensitive cell lines with wildtype TP53 genomic sequence are actually mutant. (A) CAPAN-2, MDAMB-453, MG-63, NCI-H82, and HCT116 cells were treated with either DMSO or $10 \mu \mathrm{M}$ AMG 232 for 24 hours. Total protein lysates were collected, and immunoblot analysis of MDM2, p53, and p21 expression was performed. With the exception of HCT116, expression levels of these proteins were unaffected by MDM2 inhibition, suggesting that p53 was non-functional in these lines. Additionally, MDAMB-453 lysates contained a faster migrating band, indicating truncation of p53 in that cell line. (B) Transcript sequencing of TP53 cDNA generated from CAPAN-2 and NCI-H82 showed aberrantly spliced mRNA due to a silent G $>$ T transversion in the last nucleotide of exon

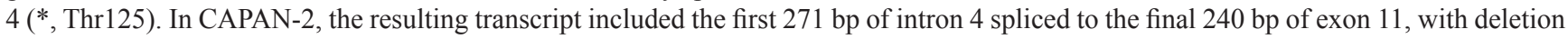
of all exons in between. In NCI-H82, the aberrant transcript deleted the last 200 bp of exon 4 (aa 59-125).

alignment against the TP53 cDNA reference sequence NM_000546 showed that a silent G > T mutation of the last nucleotide of exon 4 (Thr125Thr) affected splicing in both CAPAN-2 and NCI-H82 (Figure 3B), albeit differently. Interestingly, genomic DNA sequencing for TP53 in CAPAN-2 and NCI-H82 detected the transversion, but it was not originally identified as a mutation in these samples since it was erroneously considered a SNP (rs55863639). $\mathrm{T} 125 \mathrm{~T}$ has been reported elsewhere as a mutation known to cause errors in splicing [18-20].

With the correction of the annotation of the 4 lowexpressing " $\mathrm{p} 53^{\mathrm{WT}}$ " cell lines to $\mathrm{p} 53^{\text {Mutant }}$, the final curated set numbered 173 cell lines (Table S1). Of these, 58 lines were $\mathrm{p} 53^{\mathrm{WT}}$ and 115 were $\mathrm{p} 53^{\text {Mutant }}$. Sensitivity to MDM2 inhibition stratified absolutely according to p53 mutational status in the curated set (Figure 4).

We noted that the $\mathrm{p} 53^{\mathrm{WT}}$ cell lines as a group exhibited a wide range of $\mathrm{IC}_{50}$ values in response to MDM2 inhibition, and we therefore sought to identify additional predictors of sensitivity that might substratify these cell lines. We employed an ANOVA model which factored in tissue origin to identify genes whose expression appeared to be correlated with $\mathrm{IC}_{50}$ response (Table $\mathrm{S} 3$ ). The single most correlated gene from this model was then used to predict response in a logistic regression model. To evaluate the predictive model building process, we used leave-oneout (LOO) cross-validation and permutation testing [21]. For each permutation, the response labels were randomly shuffled within a given tissue, and then the LOO crossvalidation was repeated. We then compared the original LOO cross-validation performance ( $\%$ correct calls) to the empirical distribution of results with permuted data. The performance of the model fell at the 13th percentile of all model results based on the shuffled data. We further addressed the issue of imbalance by restricting the initial LOO-cross-validation studies to only those tissues that had both responders and non-responders. In this case, the LOO performance fell at the 67 th percentile. The results from both analyses were no different than those expected by chance, indicating that the putative stratifiers identified in 


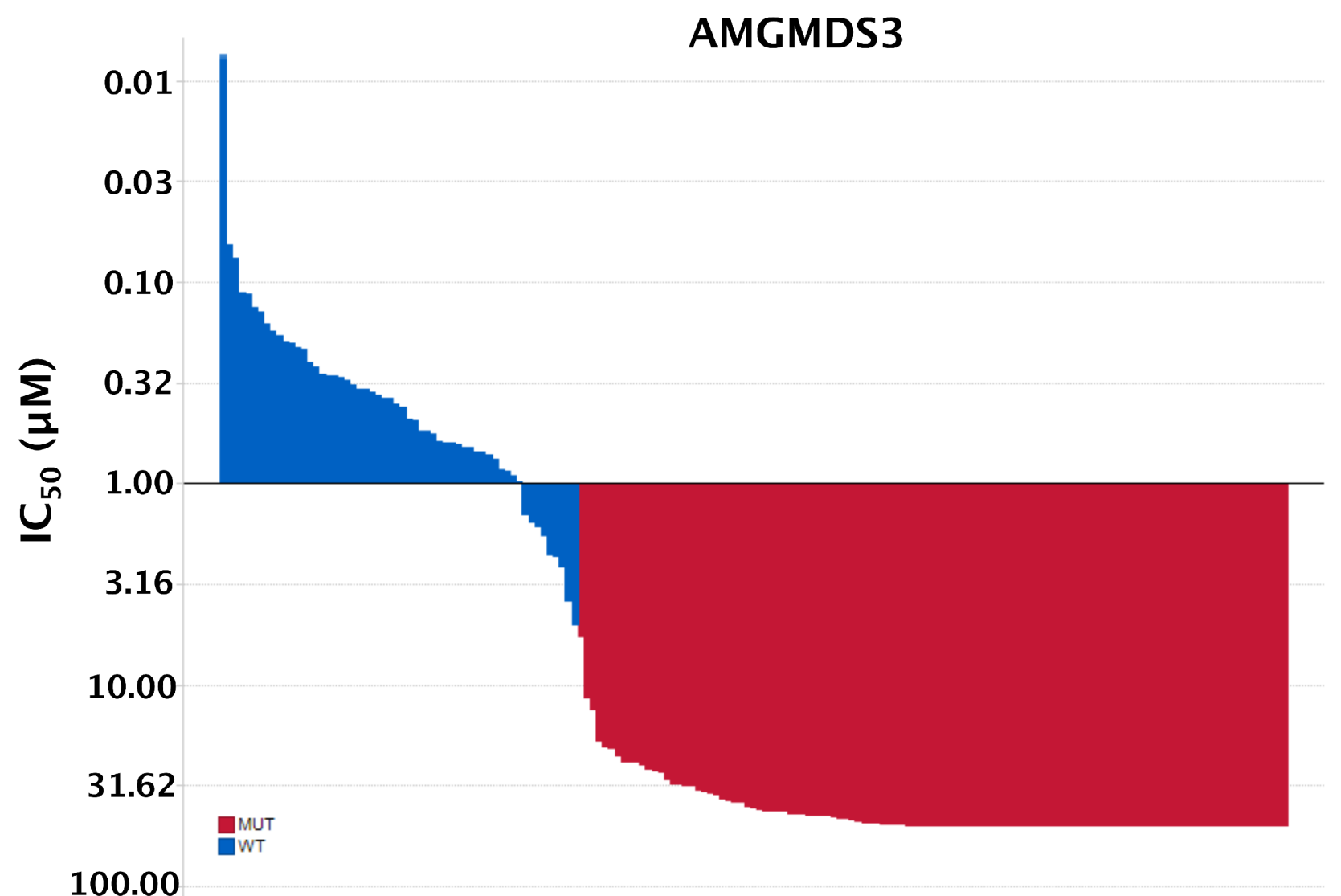

\section{Cell Lines (173)}

Figure 4: Sensitivity to MDM2 inhibition absolutely stratifies according to TP53 mutational status. 260 cell lines were originally profiled for sensitivity to MDM2 inhibitor AMGMDS3. Cell lines were removed from the panel for the following reasons: poor growth/high variance of untreated samples (26), lack of genomic DNA sample (1), misidentification (5), redundancy (22), viral infection (8), heterozygous TP53 mutation (25). The final curated set numbered 173 cell lines, of which 58 were p53 ${ }^{\mathrm{WT}}$ and 115 were p53 ${ }^{\mathrm{Mutant}}$.

our initial analysis (Table S3) were unlikely to be predictive. Furthermore, within the set of confirmed $\mathrm{p} 53^{\mathrm{WT}}$ cell lines, we also evaluated associations between AMGMDS3 sensitivity and mutation data from 64 key cancer-associated genes in the Sanger COSMIC Cell Line Project [11]. We did not identify any significant associations between sensitivity to MDM2 inhibition and mutation of these genes (Table S4).

\section{MDM2 amplification rates are likely lower than previously reported}

It has been reported that $\mathrm{p} 53^{\mathrm{WT}}$ tumor cell lines that also harbor genetically amplified $M D M 2$ are particularly sensitive to MDM2 inhibitors [22]. To determine whether this enhanced sensitivity might occur clinically, it will be critical to accurately identify which patients' tumors harbor bona fide MDM2 amplification. A frequently cited literature review reporting aggregate data compiled from multiple studies suggested that pan-cancer MDM2 amplification rates may be as high as 7\% [23], but the underlying studies used a varied array of methodologies and employed no standardized amplification copy number cutoffs. Furthermore, the reported overall amplification rate suffered from ascertainment bias, as a disproportionate number of the studies focused on tumor types previously reported to harbor high rates of $M D M 2$ amplification.

To better define the frequency of $M D M 2$ amplification in human cancer, we took advantage of the observation that MDM2 amplification and TP53 mutation are mutually exclusive phenomena [24]. This finding implies that there is no selective advantage for a tumor to have two inactivating mutations of the p53 pathway. In our analysis, we assumed that the definitive cutoff for functionally relevant $M D M 2$ amplification was the copy number associated with mutual exclusivity between MDM2 amplification and TP53 mutation. Using Oncomine NGS PowerTools (Life Technologies) to visualize the data generated by the TCGA Research Network [4], we applied this hypothesis to a pancancer analysis of 3856 TCGA tumor samples with both copy number and sequence data. After correcting for functional TP53 mutations [15], we observed that co-occurrence of MDM2 amplification and TP53 mutation decreased as the 
cutoff value for MDM2 copy number increased (Figure 5 and Table S5; $\mathrm{R}^{2}=0.9$ ). The incidence of co-occurrence for these two alterations fell to zero at MDM2 copy number $\geq 9.5$ $\left(\log _{2} \mathrm{CN}\right.$ ratio $\left.\geq 2.25\right)$, suggesting that $M D M 2$ amplification in this range was capable of functionally inactivating the p53 pathway. Applying this copy number cutoff to all tumor types represented in TCGA (Table 1) yielded MDM2 amplification rates far lower than those published previously [23], providing a more rationally defined threshold for enrolling patients into clinical trials designed to test whether MDM2 amplification affords enhanced sensitivity to MDM2 inhibition.

\section{DISCUSSION}

While MDM2 inhibitors hold great potential as cancer therapeutics, the ability to predict which tumors will respond to these agents will be critical to realizing this promise. In principle, all $\mathrm{p} 53^{\mathrm{WT}}$ tumors have the capacity to undergo cell cycle arrest upon p53 activation, and many such tumors are also expected to undergo apoptosis. In contrast, $\mathrm{p} 53^{\text {Mutant }}$ tumors would not be predicted to respond to MDM2 inhibition. Cell line sensitivity studies using MDM2 inhibitors have trended in support of these predictions, but there are numerous examples of cell lines that appear to defy these expectations.

To better understand the nature of these exceptions, we screened a panel of 260 cell lines with a potent and selective MDM2 inhibitor (AMGMDS3) to assess effects on viability. The resulting $\mathrm{IC}_{50}$ values ranged over approximately 3 orders of magnitude (Figure 1A), where the least sensitive cell lines had $\mathrm{IC}_{50}$ values $\geq 50 \mu \mathrm{M}$ (the highest concentration tested). By comparison, analogous screens performed by Garnett et al. [8] and Barretina et al. [9] using the MDM2 inhibitor, nutlin-3a, resulted in $\mathrm{IC}_{50}$ values spanning only about a 10 -fold range across the vast majority of the lines profiled (Figure $1 \mathrm{~B}$ and $1 \mathrm{C}$, respectively). The larger dynamic range afforded by AMGMDS3 over nutlin-3a is likely responsible

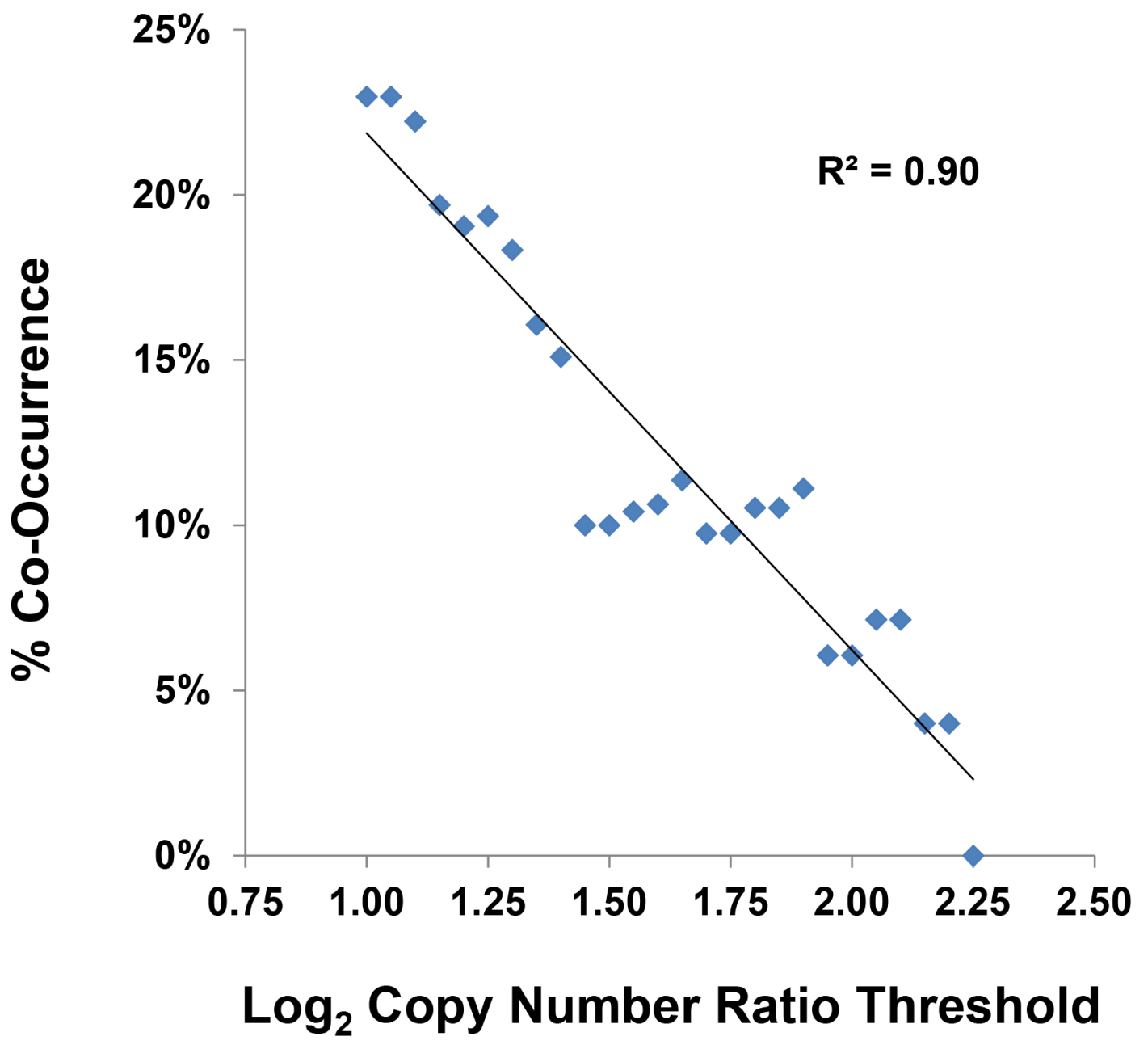

Figure 5: The threshold of functionally relevant MDM2 amplification is $\geq 9.5$ copies. Co-occurrence of $M D M 2$ amplification and TP53 mutation was analyzed using data collected by the TCGA Research Network [4] for 3856 human tumor samples. Assuming that there was no selective advantage for tumor cells to contain both inactivating mutations of the p53 pathway, the copy number threshold associated with mutual exclusivity between MDM2 amplification and TP53 mutation was a $\log _{2} \operatorname{ratio} \geq 2.25$. See also Table S5. 
Table 1: Annual U.S. incidence of MDM2-amplified tumors

\begin{tabular}{|c|c|c|c|}
\hline Tumor Type & $\begin{array}{c}M D M 2 \\
\text { Amplification }^{\dagger}\left(\mathrm{TCGA}^{\ddagger}\right)\end{array}$ & $\begin{array}{l}\text { Annual Incidence }{ }^{[30]} \\
\text { (U.S.) }\end{array}$ & $\begin{array}{l}\text { Annual U.S. Incidence with } \\
\text { MDM2 Amplification }\end{array}$ \\
\hline Liposarcoma & $64 \%(9 / 14)$ & $5,000^{[31]}$ & 3,200 \\
\hline Glioblastoma Multiforme & $7.3 \%(41 / 562)$ & $11,130^{[32]}$ & 812 \\
\hline Bladder Urothelial Carcinoma & $2.6 \%(4 / 152)$ & 74,690 & 1,942 \\
\hline Stomach Adenocarcinoma & $1.5 \%(4 / 273)$ & 22,220 & 333 \\
\hline Lung Adenocarcinoma & $0.9 \%(4 / 437)$ & $66,700^{[33]}$ & 600 \\
\hline Skin Cutaneous Melanoma & $0.7 \%(2 / 273)$ & 76,100 & 532 \\
\hline Brain Lower Grade Glioma & $0.5 \%(1 / 220)$ & $3,000^{[32,34]}$ & 15 \\
\hline Breast Invasive Carcinoma & $0.3 \%(3 / 926)$ & 235,030 & 706 \\
\hline $\begin{array}{l}\text { Ovarian Serous } \\
\text { Cystadenocarcinoma }\end{array}$ & $0.2 \%(1 / 576)$ & $13,190^{[35]}$ & 26 \\
\hline $\begin{array}{l}\text { Uterine Corpus Endometrial } \\
\text { Carcinoma }\end{array}$ & $0 \%(0 / 493)$ & 52,630 & - \\
\hline $\begin{array}{l}\text { Lung Squamous Cell } \\
\text { Carcinoma }\end{array}$ & $0 \%(0 / 387)$ & $57,170^{[33]}$ & - \\
\hline $\begin{array}{l}\text { Head and Neck Squamous } \\
\text { Cell Carcinoma }\end{array}$ & $0 \%(0 / 337)$ & 42,440 & - \\
\hline $\begin{array}{l}\text { Kidney Renal Papillary Cell } \\
\text { Carcinoma }\end{array}$ & $0 \%(0 / 117)$ & $6,400^{[36]}$ & - \\
\hline TOTAL & & & 8,166 \\
\hline
\end{tabular}

${ }^{\dagger} M D M 2 \log _{2} C N$ Ratio Cutoff $=2.25$. ${ }^{*}$ The occurrence of $M D M 2$ amplification was derived from tumor sample data generated by the TCGA Research Network [4].

for the clearer separation of $\mathrm{p} 53^{\mathrm{WT}}$ and $\mathrm{p} 53^{\text {Mutant }}$ cell lines by sensitivity. Nonetheless, even with the larger dynamic range, the initial correlation between sensitivity and p53 mutational status in our screen was not absolute. To eliminate factors potentially confounding our sensitivity analysis, we extensively curated this panel, removing cell lines that were (1) misidentified, (2) heterozygous for p53 mutation, or (3) harboring viral gene sequences known to inactivate p53. Additionally, we corrected p53 mutational status when it was misannotated and determined p53 mutational status when it was unknown. We also removed cell lines with poor growth characteristics or high coefficients of variance between replicate untreated samples. Following curation of the panel, the apparent discrepancies between sensitivity prediction and experimental observation were eliminated, revealing a perfect correlation between p53 mutational status and MDM2 inhibitor responsiveness. Based on these results, only patients with $\mathrm{p} 53^{\mathrm{WT}}$ tumors are being enrolled into clinical studies involving Amgen's MDM2 inhibitor, AMG 232.

While all tumor cell lines containing functional p53 responded to MDM2 inhibition in our screen, these lines still exhibited a 500-fold range in $\mathrm{IC}_{50}$ values from least to most sensitive. We attempted to identify the determinants of this heterogeneity by searching for genetic or expression predictors of sensitivity. Although a small number of gene expression variables were initially found to display a potential correlation between expression and sensitivity (Table S3), the fold change in expression of these genes was marginal (range $0.64-1.61$ ), and the logistic models built on this gene expression dataset were not significant. Further, there were no significant associations between response and mutational status of 64 key cancer-associated genes (Table S4). The failure to successfully predict response may have been due to the small number of $\mathrm{p} 53^{\mathrm{WT}}$ cell lines under examination or the challenges in finding such correlates in cell lines from a diverse set of tumor origins.

This report has focused on the use of MDM2 inhibitors to reduce the viability of $\mathrm{p} 53^{\mathrm{WT}}$ tumor cells. However, this is not the only context in which an MDM2 inhibitor might have clinical utility. An alternative approach has been proposed which involves the combined use of an MDM2 inhibitor with a mitotic inhibitor to treat patients harboring p53 $3^{\text {Mutant }}$ tumors $[25,26]$. Under this scenario, the MDM2 inhibitor would selectively induce reversible G1 and/or G2 cell cycle arrest in most host tissues, thus protecting them from the cytotoxic effects of chemotherapy targeting cells in M-phase. In contrast, the p53 ${ }^{\text {Mutant }}$ tumor 
cells, unaffected by MDM2 inhibition, would continue to cycle through M-phase, during which they would be susceptible to the mitotic inhibitor. In this therapeutic approach, the determinants of differential tumor response would be defined by the sensitivity of each cancer to the mitotic inhibitor, rather than to the MDM2 inhibitor.

It has been reported that the $M D M 2$-amplified subset of $\mathrm{p} 53^{\mathrm{WT}}$ tumors might be particularly sensitive to MDM2 inhibition [22]. Due to the very limited number of such cell lines in the public domain, we did not attempt to address this question in our cell panel. Instead, we chose to pursue an approach that might facilitate our ability to test this hypothesis clinically. We assumed that MDM2 amplification and TP53 mutation were mutually exclusive means for a tumor to inactivate the p53 pathway, as has been borne out in sarcomas [24]. With this hypothesis in mind, we mined the human tumor data generated by the TCGA Research Network [4] and identified a rationally derived copy number cutoff for assignment of functionally relevant MDM2 amplification. Applying this cutoff across all tumor samples within TCGA resulted in a pan-cancer MDM2 amplification rate far lower than previously published [23]. Upcoming AMG 232 clinical studies will use the same threshold to test whether $M D M 2$-amplified tumors are especially sensitive to MDM2 inhibition.

\section{METHODS}

\section{Cell lines}

Cell lines were purchased from American Type Culture Collection (ATCC), German Collection of Microorganisms and Cell Cultures (DSMZ), and Japanese Collection of Research Bioresources (JCRB). All cell lines were passaged less than 1 month prior to banking and experimentation. Unless recommended otherwise, cell lines were cultured in RPMI 1640 medium supplemented with $10 \%$ fetal bovine serum, $1 \mathrm{mM}$ sodium pyruvate, $2 \mathrm{mM}$ L-alanine, and $2 \mathrm{mM}$ L-glutamine. For authentication of cell lines at ATCC, DSMZ and JCRB, shorttandem repeat DNA typing was used.

Further authentication of a subset of cell lines was performed by Expression Analysis using the Affymetrix Genome-Wide Human SNP array 6.0. SNP genotype determination was performed using ArrayStudio. At each available SNP location, comparison was made between each of the tested samples and publically available SNP data from GlaxoSmithKline (http://www.cabig.nci.nih. gov/community/caArray_GSKdata/; [10]) or from the Wellcome Trust Sanger Institute Cancer Genome Project (http://www.sanger.ac.uk/genetics/CGP/; [11]). The total number of locations with a known genotype and the number of matched locations were tallied, and the percent genotype match was calculated for each sample. From this analysis, the best matched cell line from GSK or Wellcome Trust Sanger Institute was identified. In addition, a separate analysis was performed in the same manner, comparing the
GSK SNP genotype data for all cell lines in the panel to the Wellcome Trust Sanger Institute CGP SNP data in order to identify any synonymous or misidentified cell lines.

\section{Cell proliferation assay}

Inhibition of cell proliferation was measured by Eurofins Panlabs (formerly Ricerca Biosciences). Cells were seeded into 384-well plates. After 24 hours, a 10-point titration of MDM2 inhibitor was added to the wells in a final DMSO concentration of $0.1 \%$. After 72-hour treatment, cells were fixed and stained with dye to allow visualization of nuclei. Automated fluorescence microscopy was carried out using a GE Healthcare IN Cell Analyzer 1000 , and images were collected with a $4 \mathrm{X}$ objective.

Cell proliferation was measured by incorporation of nuclear dye to determine the relative cell count. To calculate the effect of compound treatment on cell proliferation, the data were transformed to percent of control (POC) using the following formula: $\mathrm{POC}=$ relative cell count (compound wells)/relative cell count (vehicle wells) $\times 100 . \mathrm{IC}_{50}$ values were derived using nonlinear regression to fit data to a sigmoidal 4-point, 4-parameter one-site dose response model, where: y (fit) $=\mathrm{A}+\left[(\mathrm{B}-\mathrm{A}) /\left(1+\left((\mathrm{C} / \mathrm{x})^{\wedge} \mathrm{D}\right)\right)\right]$. Relative cell count $\mathrm{IC}_{50}$ was the inhibitor concentration that produced $50 \%$ of the cell proliferation inhibitory response or $50 \%$ cytotoxicity level, relative to DMSO control.

\section{Genomic analysis of TP53}

Genomic DNA was extracted from frozen cell pellets of each tumor cell line according to the DNeasy 96 kit protocol (QIAGEN) and sequenced for the presence of mutations in TP53, either using 454 sequencing (Roche Diagnostics) or $\mathrm{WAVE}^{\circledR}$ Mutation Detection System and SURVEYOR ${ }^{\circledR}$ Nuclease technology (Transgenomic, Inc.; [27]).

Additional data regarding the p53 mutational status of tumor cell lines in the panel were obtained from the Wellcome Trust Sanger Institute Catalogue of Somatic Mutations in Cancer (COSMIC v44-62 releases), http:// www.sanger.ac.uk/cosmic [11, 28]; the IARC TP53 database (version R15), p53.iarc.fr/TP53GeneVariations.aspx [29], and the TP53 Mutation Database, http://www.p53.fr [18].

\section{Quantitative PCR screening for p53-inactivating viral DNA sequences}

Primers to detect viral DNA sequences for human papillomavirus E6 from high- risk variants 16, 18, 31, 33, and 45, as well as simian virus 40 large $\mathrm{T}$ antigen and adenovirus E1B were designed using Primer Express software and synthesized by Integrated DNA Technologies (Table S2). Genomic DNA samples from human tumor cell lines were screened by quantitative RT-PCR using an Applied Biosystems Prism 7900HT instrument. 


\section{Sequencing of TP53 transcript}

Total RNA was isolated from human tumor cell lines according to the RNeasy Mini Kit protocol (QIAGEN). 3'RACE-ready cDNA was generated according to the SMARTer RACE cDNA Amplification Kit protocol (Clontech). 3' RACE PCR, followed by additional nested PCR, was performed to amplify TP53 cDNA sequence from each of the samples. PCR products were electrophoresed on $1 \%$ agarose gels, and the desired PCR products were isolated, purified, cloned, and sequenced. Sequences from the cDNA clones were aligned against the TP53 cDNA reference sequence NM_000546 using Vector $\mathrm{NTI}^{\circledR}$ software (Life Technologies).

\section{Immunoblot analysis}

Cells were seeded at subconfluent densities in 6-well plates and incubated overnight at $37^{\circ} \mathrm{C}$ and $5 \%$ $\mathrm{CO}_{2}$. The following day, either DMSO or AMG 232 was added to appropriate wells at a final concentration of $0.1 \%$ DMSO or $10 \mu \mathrm{M}$, respectively. After incubation for 24 hours at $37^{\circ} \mathrm{C}$ and $5 \% \mathrm{CO}_{2}$, protein lysates were collected, electrophoresed on 10\% Bis-Tris NuPAGE gels (Life Technologies), and transferred to Invitrolon PVDF membranes (Life Technologies). Following transfer, the membranes were blocked and incubated with primary antibodies against p53 (DO-1; Calbiochem), MDM2 (BD Pharmingen), p21 (R\&D Systems), or $\beta$-actin-HRP (Sigma). For detection, the membranes were incubated with species-specific secondary antibodies conjugated to HRP. Luminescent signal was developed using ECL Plus reagent (GE Healthcare) and the membranes were exposed to film.

\section{Association analysis}

The vast majority of the gene expression data was obtained from the GlaxoSmithKline Cancer Cell Line Genomic Profiling dataset [10]. For cell lines without publicly available data, analogous data were obtained from Eurofins Panlabs (formerly Ricerca Biosciences). Gene expression data were generated using the Affymetrix HGU133 Plus 2.0 microarray platform. RMA-normalized gene expression data from Greshock et al. [10] and from Eurofins Panlabs were combined using a per probe set linear batch correction based on 20 cell lines profiled in both data sets.

Association analyses were performed to attempt to identify additional predictors of sensitivity. Response to MDM2 inhibition was categorized as true if the $\mathrm{IC}_{50}$ for a cell line was in the lower quartile of $\mathrm{IC}_{50}$ values among all cell lines; otherwise it was set to false. An ANOVA model including a factor for tumor origin was used as a feature selection step to identify genes whose expression were highly correlated with $\mathrm{IC}_{50}$ response. The single most correlated gene from the feature selection step was used in a logistic regression model to predict response given the gene expression value and tissue source of a new cell line. If the model-based probability of response was predicted to be greater than 0.5 , the new cell line was classified as a responder; otherwise it was classified as a non-responder. The leave-one-out (LOO) cross-validation procedure recommended by Simon et al. [21] was used to estimate the performance of the entire model building process, including feature selection. A single sample was left out, and the remainder was used to identify a gene expression variable and to build a predictive model. The predictive model was used to predict response (true/false) of the sample that was left out. The process was repeated, leaving each sample out in turn. At the end of the LOO cross-validation, the predictions were compared to known results. The performance of the LOO cross-validation was evaluated based on permutation testing. For each permutation, the response labels were randomly shuffled within a given tissue, and then the LOO cross-validation was repeated. We then compared the original LOO cross-validation performance ( $\%$ correct calls) to the empirical distribution of results with permuted data. We further addressed the issue of imbalance by restricting the initial LOO-cross-validation studies to only those tissues that had both responders and non-responders.

Mutation data were collected from the Wellcome Trust Sanger Institute COSMIC Cell Line Project (http:// www.cancer.sanger.ac.uk/cancergenome/projects/cell_lines/; v61 release) [11]. The mutation status of 64 key cancerassociated genes was available for 51 of the $58 \mathrm{p} 53^{\mathrm{WT}}$ cell lines in the panel. There were 25 genes for which at least one cell line was called mutant. We evaluated associations between response to MDM2 inhibition and mutation status using the "aov" function in R. Test $p$-values were adjusted using the R "p.adjust" function with method = "BH".

\section{ACKNOWLEDGMENTS}

We would like to thank Sharon Zhao and Kira Misura for their contributions to the analysis of the MDM2 inhibitor sensitivity and sequence data from the 260-cell line panel; Gene Cutler for his assistance with association analyses; Usha Warrior and Jameel Shah (Eurofins Panlabs), as well as Seth Sadis (Compendia Bioscience) for providing reagents and data which allowed us to thoroughly curate the cell line dataset. All authors are shareholders and current (A.S., S.C., C.S. and M.B.) or former (E.C. and J.O.) employees of Amgen, Inc.

\section{REFERENCES}

1. Levine AJ. p53, the cellular gatekeeper for growth and division. Cell. 1997; 88:323-331.

2. Vogelstein B, Lane D, Levine AJ. Surfing the p53 network. Nature. 2000; 408:307-310.

3. Levine AJ, Oren M. The first 30 years of p53: growing ever more complex. Nature reviews Cancer. 2009; 9:749-758. 
4. The Cancer Genome Atlas Research Network TCGA

5. Vassilev LT, Vu BT, Graves B, Carvajal D, Podlaski F, Filipovic Z, Kong N, Kammlott U, Lukacs C, Klein C, Fotouhi N, Liu EA. In vivo activation of the p53 pathway by small-molecule antagonists of MDM2. Science. 2004; 303:844-848

6. Sun D, Li Z, Rew Y, Gribble M, Bartberger MD, Beck HP, Canon J, Chen A, Chen X, Chow D, Deignan J, Duquette J, Eksterowicz J, Fisher B, Fox BM, Fu J, et al. Discovery of AMG 232, a Potent, Selective, and Orally Bioavailable MDM2-p53 Inhibitor in Clinical Development. Journal of medicinal chemistry. 2014; 57:1454-1472.

7. Tovar C, Graves B, Packman K, Filipovic Z, Xia BH, Tardell C, Garrido R, Lee E, Kolinsky K, To KH, Linn M, Podlaski F, Wovkulich P, Vu B, Vassilev LT. MDM2 Small-Molecule Antagonist RG7112 Activates p53 Signaling and Regresses Human Tumors in Preclinical Cancer Models. Cancer Res. 2013; 73:2587-2597.

8. Garnett MJ, Edelman EJ, Heidorn SJ, Greenman CD, Dastur A, Lau KW, Greninger P, Thompson IR, Luo X, Soares J, Liu Q, Iorio F, Surdez D, Chen L, Milano RJ, Bignell GR, et al. Systematic identification of genomic markers of drug sensitivity in cancer cells. Nature. 2012; 483:570-575.

9. Barretina J, Caponigro G, Stransky N, Venkatesan K, Margolin AA, Kim S, Wilson CJ, Lehar J, Kryukov GV, Sonkin D, Reddy A, Liu M, Murray L, Berger MF, Monahan JE, Morais P, et al. The Cancer Cell Line Encyclopedia enables predictive modelling of anticancer drug sensitivity. Nature. 2012; 483:603-607.

10. Greshock J, Bachman KE, Degenhardt YY, Jing J, Wen YH, Eastman S, McNeil E, Moy C, Wegrzyn R, Auger K, Hardwicke MA, Wooster R. Molecular target class is predictive of in vitro response profile. Cancer Res. 2010; 70:3677-3686.

11. Forbes SA, Bindal N, Bamford S, Cole C, Kok CY, Beare D, Jia M, Shepherd R, Leung K, Menzies A, Teague JW, Campbell PJ, Stratton MR, Futreal PA. COSMIC: mining complete cancer genomes in the Catalogue of Somatic Mutations in Cancer. Nucleic Acids Res. 2011; 39:D945-950.

12. Scheffner M, Werness BA, Huibregtse JM, Levine AJ, Howley PM. The E6 oncoprotein encoded by human papillomavirus types 16 and 18 promotes the degradation of $\mathrm{p} 53$. Cell. 1990; 63:1129-1136.

13. Thomas M, Pim D, Banks L. The role of the E6-p53 interaction in the molecular pathogenesis of HPV. Oncogene. 1999; 18:7690-7700.

14. Smith ML, Chen IT, Zhan Q, O’Connor PM, Fornace AJ Jr. Involvement of the p53 tumor suppressor in repair of u.v.type DNA damage. Oncogene. 1995; 10:1053-1059.

15. Kato S, Han SY, Liu W, Otsuka K, Shibata H, Kanamaru R, Ishioka C. Understanding the function-structure and function-mutation relationships of p53 tumor suppressor protein by high-resolution missense mutation analysis. Proc Natl Acad Sci U S A. 2003; 100:8424-8429.

16. Wasielewski M, Elstrodt F, Klijn JG, Berns EM, Schutte M. Thirteen new p53 gene mutants identified among 41 human breast cancer cell lines. Breast cancer research and treatment. 2006; 99:97-101.

17. Chandar N, Billig B, McMaster J, Novak J. Inactivation of p53 gene in human and murine osteosarcoma cells. Br J Cancer. 1992; 65:208-214.

18. Edlund K, Larsson O, Ameur A, Bunikis I, Gyllensten U, Leroy B, Sundstrom M, Micke P, Botling J, Soussi T. Datadriven unbiased curation of the TP53 tumor suppressor gene mutation database and validation by ultradeep sequencing of human tumors. Proc Natl Acad Sci U S A. 2012; 109:9551-9556.

19. Varley JM, Attwooll C, White G, McGown G, Thorncroft M, Kelsey AM, Greaves M, Boyle J, Birch JM. Characterization of germline TP53 splicing mutations and their genetic and functional analysis. Oncogene. 2001; 20:2647-2654.

20. Soussi T. TP53 Mutations in Human Cancer: Database Reassessment and Prospects for the Next Decade. In: George K, ed. Advances in cancer research: Academic Press. 2011; pp. 107-139.

21. Simon R, Radmacher MD, Dobbin K, McShane LM. Pitfalls in the use of DNA microarray data for diagnostic and prognostic classification. Journal of the National Cancer Institute. 2003; 95:14-18.

22. Tovar C, Rosinski J, Filipovic Z, Higgins B, Kolinsky K, Hilton $\mathrm{H}$, Zhao X, Vu BT, Qing W, Packman K, Myklebost O, Heimbrook DC, Vassilev LT. Small-molecule MDM2 antagonists reveal aberrant p53 signaling in cancer: implications for therapy. Proc Natl Acad Sci U S A. 2006; 103:1888-1893.

23. Momand J, Jung D, Wilczynski S, Niland J. The MDM2 gene amplification database. Nucleic Acids Res. 1998; 26:3453-3459.

24. Leach FS, Tokino T, Meltzer P, Burrell M, Oliner JD, Smith S, Hill DE, Sidransky D, Kinzler KW, Vogelstein B. p Mutation and MDM2 amplification in human soft tissue sarcomas. Cancer Res. 1993; 53:2231-2234.

25. Apontes $\mathrm{P}$, Leontieva OV, Demidenko ZN, Li F, Blagosklonny MV. Exploring long-term protection of normal human fibroblasts and epithelial cells from chemotherapy in cell culture. Oncotarget. 2011; 2:222-233.

26. Carvajal D, Tovar C, Yang H, Vu BT, Heimbrook DC, Vassilev LT. Activation of p53 by MDM2 antagonists can protect proliferating cells from mitotic inhibitors. Cancer Res. 2005; 65:1918-1924.

27. Janne PA, Borras AM, Kuang Y, Rogers AM, Joshi VA, Liyanage H, Lindeman N, Lee JC, Halmos B, Maher EA, Distel RJ, Meyerson M, Johnson BE. A rapid and sensitive enzymatic method for epidermal growth factor receptor mutation screening. Clinical cancer research: an official 
journal of the American Association for Cancer Research. 2006; 12:751-758.

28. Forbes SA, Bhamra G, Bamford S, Dawson E, Kok C, Clements J, Menzies A, Teague JW, Futreal PA, Stratton MR. The Catalogue of Somatic Mutations in Cancer (COSMIC). Current protocols in human genetics/editorial board. Jonathan L Haines [et al]. 2008; Chapter 10:Unit 10-11.

29. Petitjean A, Mathe E, Kato S, Ishioka C, Tavtigian SV, Hainaut P, Olivier M. Impact of mutant p53 functional properties on TP53 mutation patterns and tumor phenotype: lessons from recent developments in the IARC TP53 database. Human mutation. 2007; 28:622-629.

30. American Cancer Society. Cancer Facts \& Figures 2014. Atlanta: American Cancer Society; 2014.

31. http://emedicine.medscape.com/article/1102007-overview \#a0199.

32. Dolecek TA, Propp JM, Stroup NE, Kruchko C. CBTRUS Statistical Report: Primary Brain and Central Nervous
System Tumors Diagnosed in the United States in 20052009. Neuro-Oncology. 2012; 14:v1-v49.

33. http://www.lungcancerresearchfoundation.org/lung-cancertypes/.

34. Pouratian N, Asthagiri A, Jagannathan J, Shaffrey ME, Schiff D. Surgery Insight: the role of surgery in the management of low-grade gliomas. Nat Clin Pract Neuro. 2007; $3: 628-639$

35. Levanon K, Crum C, Drapkin R. New insights into the pathogenesis of serous ovarian cancer and its clinical impact. Journal of clinical oncology: official journal of the American Society of Clinical Oncology. 2008; 26:5284-5293.

36. Yang XJ, Tan M-H, Kim HL, Ditlev JA, Betten MW, Png CE, Kort EJ, Futami K, Furge KA, Takahashi M, Kanayama H-o, Tan PH, Teh BS, Luan C, Wang K, Pins M, et al. A Molecular Classification of Papillary Renal Cell Carcinoma. Cancer Res. 2005; 65:5628-5637. 